\title{
The human haptoglobin gene promoter: interleukin-6-responsive elements interact with a DNA-binding protein induced by interleukin-6
}

\section{Salvatore Oliviero and Riccardo Cortese}

\author{
European Molecular Biology Laboratory, Meyerhofstrasse 1, 6900 \\ Heidelberg, FRG \\ Communicated by R.Cortese
}

Transcription of the human haptoglobin (Hp) gene is induced by interleukin-6 (IL-6) in the human hepatoma cell line Hep3B. Cis-acting elements responsible for this response are localized within the first $186 \mathrm{bp}$ of the 5 '-flanking region. Site-specific mutants of the $\mathrm{Hp}$ promoter fused to the chloramphenicol acetyl transferase (CAT) gene were analysed by transient transfection into uninduced and IL-6-treated Hep3B cells. We identified three regions, $\mathrm{A}, \mathrm{B}$ and $\mathrm{C}$, defined by mutation, which are important for the IL-6 response. Band shift experiments using nuclear extracts from untreated or IL-6-treated cells revealed the presence of IL-6-inducible DNA binding activities when DNA fragments containing the $\mathrm{A}$ or the $\mathrm{C}$ sequences were used. Competition experiments showed that both sequences bind to the same nuclear factors. Polymers of oligonucleotides containing either the $A$ or the $C$ regions confer IL- 6 responsiveness to a truncated SV40 promoter. The B region forms several complexes with specific DNA-binding proteins different from those which bind to the $A$ and $C$ region. The $B$ region complexes are identical in nuclear extracts from IL-6-treated and untreated cells. While important for IL-6 induction in the context of the haptoglobin promoter, the B site does not confer IL-6 inducibility to the SV40 promoter. Our results indicate that the IL-6 response of the haptoglobin promoter is dependent on the presence of multiple, partly redundant, cis-acting elements.

Key words: haptoglobin/interleukin-6/inflammation

\section{Introduction}

Several lines of evidence indicate that nuclear proteins which bind to cis-acting elements of gene promoters are responsible for the differential expression and induction of specific genes in different tissues (Ptashne, 1988).

Many genes specifically expressed in hepatocytes have been characterized in detail (for review, see De Simone and Cortese, 1989). A subset of liver-specific genes are subject to modulation of their transcriptional rate during inflammation. This property is at the root of the so-called acute phase reaction (AP reaction), recently reviewed (Kushner, 1982; Koj, 1987; Ciliberto, 1988; Fey and Gauldie, 1988). Apparently several cytokines, synthesized by macrophages and other cells, are responsible for this effect. The cytokines presumably interact with specific receptors present on the surface of hepatocytes (Baumann et al., 1983a,b, 1984, 1986, 1987a,b; Koj et al., 1984; Ramadori et al., 1985;
Woloski and Fuller, 1985; Perlmutter et al., 1986; Gauldie et al., 1987). Not much is known about the mechanism of this gene regulation. Recently however some insight into this phenomenon has been gained from experiments performed with cultured cell lines.

The AP response can be reproduced in vitro by treatment of the hepatoma cell line Hep3B (Knowles et al., 1981) with conditioned medium of activated monocytes (MoCM) (Darlington et al., 1986). Recently several groups have identified the monokine interleukin-6 (IL-6) as one of the most important mediators of the liver AP response (Gauldie et al., 1987). IL-6 is the new name given to an activity previously called interferon- $\beta 2$ or BSF- 2 . It is a $26-\mathrm{kd}$ protein with pleiotropic functions (reviewed by Billiau, 1987; Seghal et al., 1987; Kishimoto and Hirano, 1988; Wong and Clark, 1988). We have shown that IL-6 regulates the transcriptional activation of the human haptoglobin ( $\mathrm{Hp})$, complement factor B (Cf B), alpha-1-acid glycoprotein (AGP) and C-reactive protein (CRP) genes in the hepatoma cell line Hep3B (Morrone et al., 1988).

By means of transfection experiments we have shown that the -186 to +30 segment of the $\mathrm{Hp}$ promoter is able to confer cell-specific expression and IL-6 responsiveness to a reporter gene (Oliviero et al., 1987; Morrone et al., 1988). These data indicate that the haptoglobin response to IL-6 induction could be caused by an IL-6-dependent change in the concentration and/or in the activity of trans-acting transcriptional factors which bind to Hp promoter elements. Here we have analysed the Hp promoter by site-directed mutagenesis and identified cis-acting elements that are responsible for the activation of transcription and for the response to IL-6. We also identify a DNA-binding protein whose activity is induced by IL-6.

\section{Results}

Expression of the cloned Hp promoter in Hep3B cells In a previous study it was shown that the promoter of the human haptoglobin gene, from position -186 to +1 was capable of directing transcription in HepG2 cells but not in HeLa cells, thus showing that in this DNA segment there are hepatoma-specific cis-acting elements. In contrast, the same constructs were poorly expressed in another hepatoma cell line, Hep3B. In the latter, however, Hp transcription could be induced by treatment of the cells with IL-6 (Oliviero et al., 1987; Morrone et al., 1988).

In order to analyse the promoter elements contained in this DNA sequence in greater detail, site-directed mutagenesis of the $\mathrm{Hp} 5^{\prime}$-flanking region was performed. In each mutant six bases of the $\mathrm{Hp}$ promoter sequence were replaced by the sequence GATATC, the EcoRV restriction site (Figure 1). Ten different mutants, spanning the region -162 to -48 with respect to the RNA start site, were constructed. The mutants were designed by numbers 


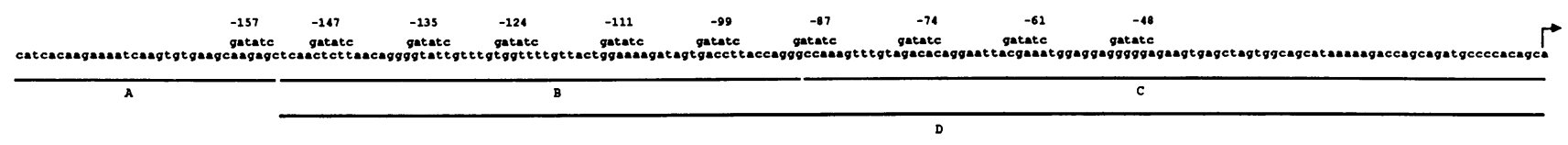

Fig. 1. Sequence of the $5^{\prime}$-flanking region of the $\mathrm{Hp}$ gene from -186 to +1 . The different mutants are indicated by numbers corresponding to the $3^{\prime}$ end of the mutated region. Each mutant carries the corresponding substitutions as indicated. In the bottom part the DNA segments used as probes for the in vitro experiments are indicated.

\begin{tabular}{llcc}
\hline \multicolumn{4}{l}{ Table I. Expression of Hp-CAT } \\
\hline Templates & Hep3B & Hep3B + IL-6 & Induction ratio \\
\hline wt & 1.0 & 27.6 & 27.6 \\
M-157 & 0.6 & 6.0 & 10.0 \\
M-147 & 1.2 & 25.5 & 21.2 \\
M-135 & 1.5 & 40.0 & 26.6 \\
M-124 & 1.8 & 37.0 & 20.5 \\
M-111 & 1.0 & 4.5 & 4.5 \\
M-99 & 1.0 & 30.0 & 30.0 \\
M-87 & 1.0 & 30.0 & 30.0 \\
M-74 & 0.6 & 15.0 & 25.0 \\
M-61 & 0.4 & 1.5 & 3.7 \\
M-48 & 0.8 & 20.4 & 25.5 \\
\hline
\end{tabular}

The value of 1 corresponds to the expression of the wild-type Hp promoter in uninduced Hep3B cells.

corresponding to their $3^{\prime}$ boundaries. The mutant DNAs were analysed by transient transfection into IL-6-treated or untreated Hep3B cells, to identify IL-6-responsive elements. Transcriptional activity, measured as the rate of chloramphenicol acetylation, was compared with that of the wild-type promoter. The transcriptional activities of the transfected plasmids were normalized by comparison with an internal control obtained by co-transfection of a plasmid (RSV-LUC) containing the Rous sarcoma virus promoter sequences fused to the coding sequence of the firefly luciferase gene (DeWet et al., 1987).

The basal level of expression of the haptoglobinchloramphenicol acetyl transferase (CAT) fusion containing the wild-type sequence in Hep3B cells is very low, making it impossible to evaluate accurately the effect of the various mutations. Transcription is induced $>20$-fold by treatment with IL-6 (Table I). Three mutations show impaired inducibility: M-157 is only induced 10 -fold while $\mathrm{M}-111$ and M-61 are more severely affected (Table I). These mutations define three regions, A, B and C, apparently important for expression of the haptoglobin promoter in the presence of IL-6.

\section{Nuclear factors binding to the $A, B$ and $C$ domain of the Hp promoter}

The functional assays described in the previous section suggest that the $\mathrm{A}, \mathrm{B}$ and $\mathrm{C}$ regions in the Hp promoter are important for Hp promoter activity and for full responsiveness to IL-6. In order to identify trans-acting factors possibly repsonsible for such transcriptional effects, in vitro binding studies were performed by gel retardation analysis using nuclear extracts from Hep3B cells. As probes we used end-labelled DNA fragments derived from the $\mathrm{Hp}$ promoter (as indicated in Figure 1). Several nucleoprotein-DNA complexes were observed with all three fragments. Comparison of the shifted bands obtained using fragment $\mathrm{C}$ with extracts from untreated (lane a) and IL-6-treated Hep3B (lane b) reveals a difference: the appearance in the

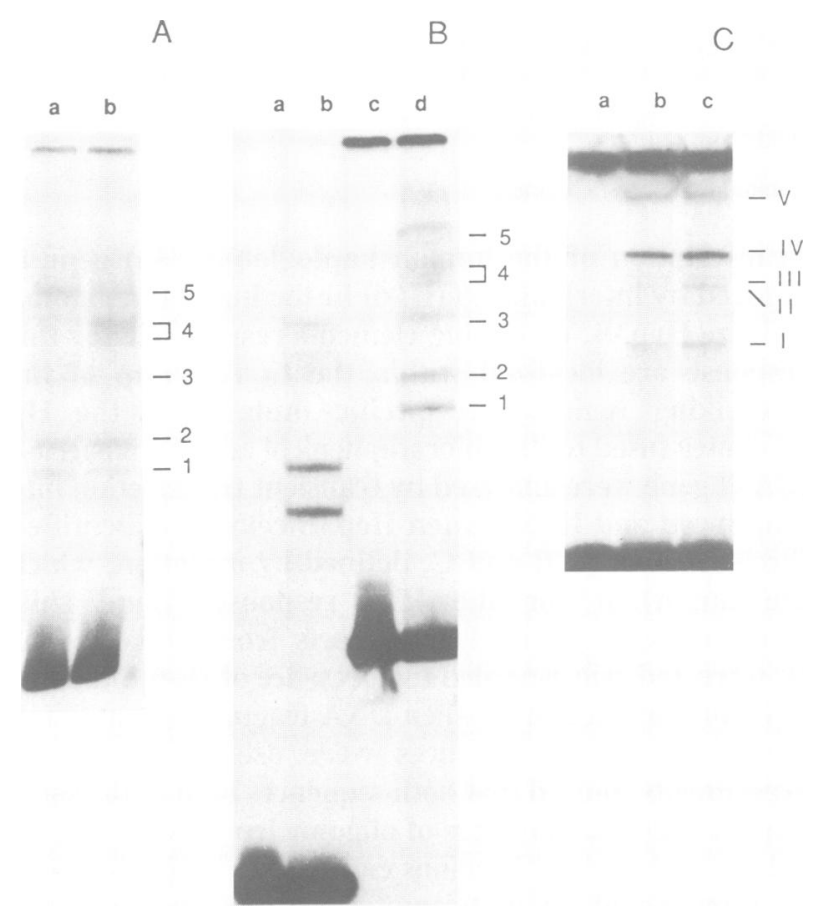

Fig. 2. Gel retardation experiments. In panel $\mathrm{A}$ the $\mathrm{Hp} \mathrm{C}$ fragment (see Figure 1) was used as probe. Lane a, the probe was incubated with extract of unstimulated Hep3B cells and lane b, Hep3B, after IL-6 treatment. Numbers indicate the different retarded bands formed. In panel B the $\mathrm{Hp} \mathrm{A}$ (lanes $\mathbf{a}$ and $\mathbf{b}$ ) and the $\mathrm{Hp} \mathrm{C}$ fragments (lanes c and d) were used as probes. Lanes a and $\mathbf{c}$, no extract; lanes $\mathbf{c}$ and d), extract of IL-6-treated Hep3B cells. In panel C the Hp B fragment was used as a probe. Lane b, extract from unstimulated Hep3B cells; lane c, from IL-6-treated Hep3B. Roman numerals indicate the different retarded band formed.

latter of new complexes, indicated in the figure as complex 4. In some experiments complex 4 was resolved in several closely-spaced bands. A similar pattern of shifted bands was obtained with the A probe, containing the A domain, defined by the -157 mutation, both with induced (compare Figure 2B, lane b with lane d) and uninduced Hep3B nuclear extract (data not shown). Note that the A fragment is much shorter than the $\mathrm{C}$ fragment. The distribution of complexes observed appears similar, but all the $\mathrm{C}$ complexes migrate correspondingly more slowly than the A complexes.

When the B fragment ( -153 to -87$)$ was used as a probe, a different pattern of retarded bands appeared (Figure 2C). We were able to observe these complexes (indicated with Roman numbers I-V) only when salmon sperm DNA was omitted from the reaction mixture. The pattern of retarded bands obtained with induced and uninduced extracts is identical (compare Figure 2C, lane b with lane $\mathrm{c}$ ).

To further characterize the nature of the DNA - protein complexes, we have performed additional gel shift experiments using wild-type $\mathrm{Hp}$ promoter fragments as radioactive probes in the presence of a variety of synthetic 
Table II. Oligonucleotides used in this study
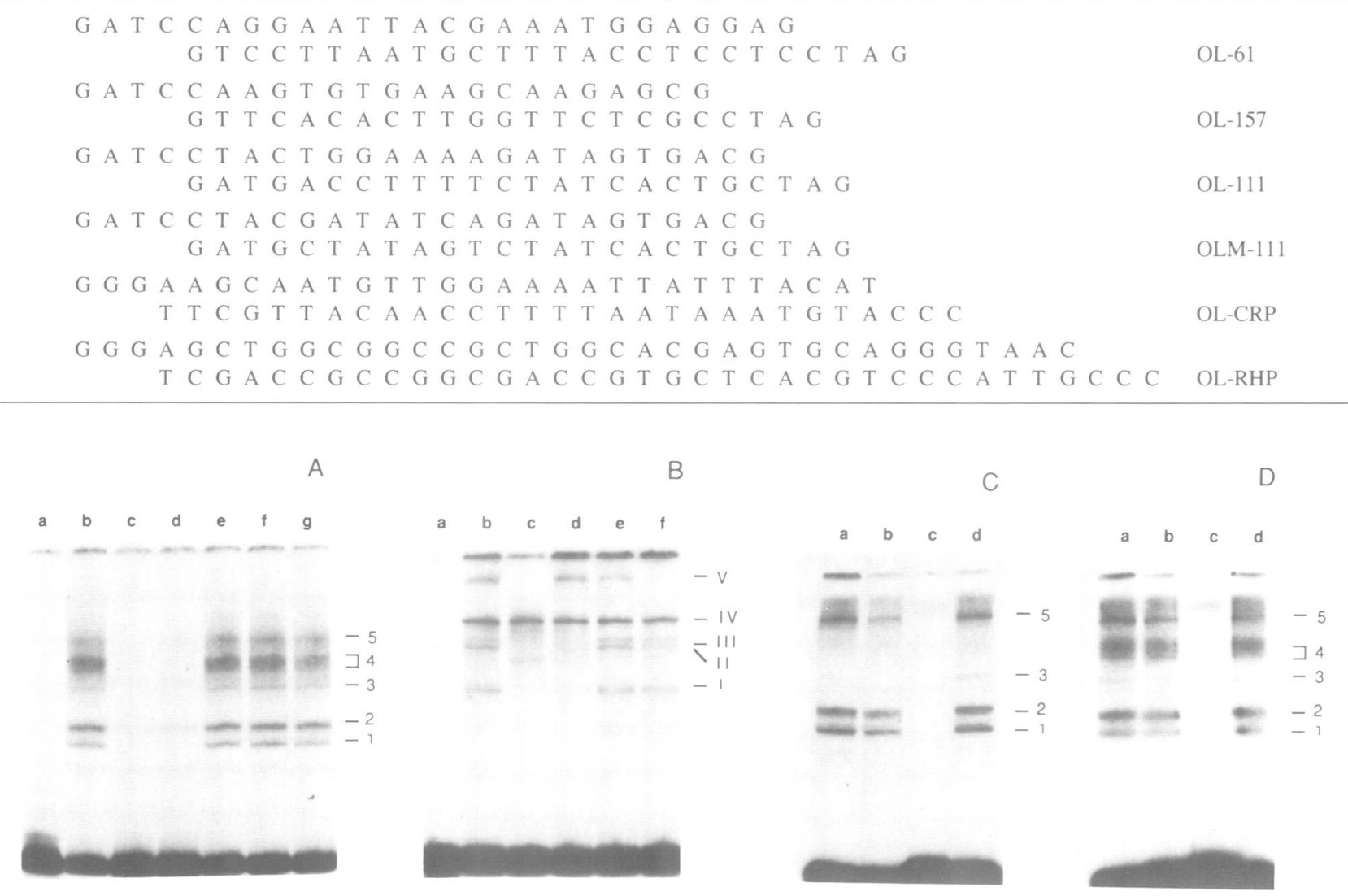

Fig. 3. Gel retardation experiments with specific competitors. Panel A: the Hp C fragment was used as a probe and incubated with IL-6-treated Hep3B nuclear extract in the presence of 400-fold molar excess of specific cold competitor. Lane a. no extract: lane b. probe alone: lane c. with OL-61 (see Table I): lane d. with OL-157: lane e, with OL-111: lane f. with OL-RBP: lane g. with OL-CRP. Panel B: the Hp B fragment was used as a probe. Lane a. no extract; lane b. probe alone: lane c. with OL-111. lane d. with OLM-111: lane e, with OL-61: lane f. with OL-CRP. In panel C different probes were incubated with Hep3B nuclear extract in the presence of salmon sperm DNA as competitor. Lane a. the Hp D fragment (see Figure 1): lane b the Hp D fragment from the HpM-48 mutant: lane c. the $\mathrm{Hp} \mathrm{D}$ fragment from the $\mathrm{HpM}-61 \mathrm{mutant}$ : lane d. the Hp D fragment from the HpM-74 mutant. Panel D. the same as in panel C but with IL-6-treated Hep3B nuclear extract.

double-stranded oligonucleotides used as competitors (listed in Table II). In Figure 3A DNA fragment $C$ was used as such (lane $b$ ) and in the presence of 400 -fold molar excess of different oligonucleotides (lanes c,d,e,f and $g$ ). The oligonucleotide OL-61, containing 22 bases of the $\mathrm{Hp}$ promoter centred at the Hp-61 sequence (Table II) competes for all the complexes (Figure 3A, lane c). Also OL-157 (18 bases centred at the Hp-157 sequence, Table II) is an efficient competitor of all complexes (Figure $3 \mathrm{~A}$, lane d). This result indicates that the same proteins interact with the $\mathrm{A}$ and the $\mathrm{C}$ domain. The oligonucleotide OL-111 (19 bases centred at the Hp-111 sequence, Table II), OL-RBP (Paonessa et al., 1988) or OL-CRP (Arcone et al., 1988) (see Table II) did not compete for any of the complexes. Identical results were obtained using fragment $\mathrm{A}$ (Figure 1) as a radioactive probe (data not shown). These results indicate that fragment $\mathrm{A}$ and $\mathrm{C}$ interact with the same DNA-binding proteins and that the different gel mobility of these complexes is due to the different length of the DNA fragments. This was confirmed using as probes oligonucleotides containing the $\mathrm{A}$ and the $\mathrm{C}$ sequences (data not shown).

Of the multiple retarded bands observed with fragment B (Figure 3B, lane b), complex IV could not be competed by any oligonucleotide. Complexes V, III, II and I were competed by OL-111 (lane c). Of these only complex $\mathrm{V}$ was not competed by OLM-111 (lane d), an oligo centred at the Hp-111 position, like OL-111, but carrying the M-111 mutation (see Table II). OL-61 (lane e) and OL-157 (not shown) did not compete for the formation of any of the complexes. Interestingly, specific inhibition of the formation of complex $\mathrm{V}$ (lane f) was obtained with an oligonucleotide derived from the promoter of the AP responsive gene coding for the C-reactive protein (OL-CRP, Table II, see also Arcone et al., 1988).

In order to test whether a direct correlation between binding activity and expression could be observed, we performed gel retardation experiments using as probes $\mathrm{Hp}$ promoter DNA fragments carrying the mutations used in the transfection experiments. As shown in Figure 3C (with uninduced extract) and 3D (with IL-6-induced extract), the M-61 mutation interferes with the formation of all the complexes, although an ill-defined low mobility band which has not been characterized is still present (Figure 3C, lane $\mathrm{c}$ and 3D, lane c). Similar results were obtained with mutant M-157 (not shown). The presence of the mutations M-48 or M-74 (lanes b and d) in the DNA fragments used did not interfere with the formation of any of the complexes. Similar results were obtained with mutant -157 (data not shown).

These results indicate that the same interactions occur at the domain $\mathrm{A}$ and domain $\mathrm{C}$ of the Hp promoter, and that 
A

$$
\text { Hp } \quad-186 /-87
$$

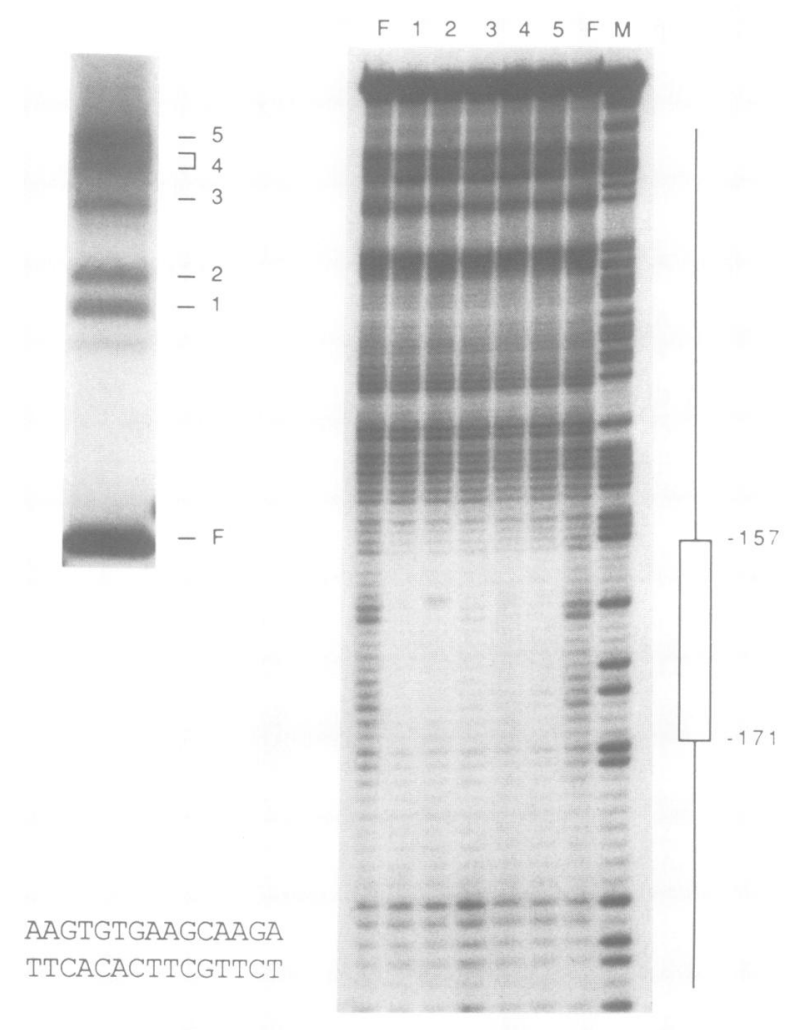

Hp $\quad-153 /-20$

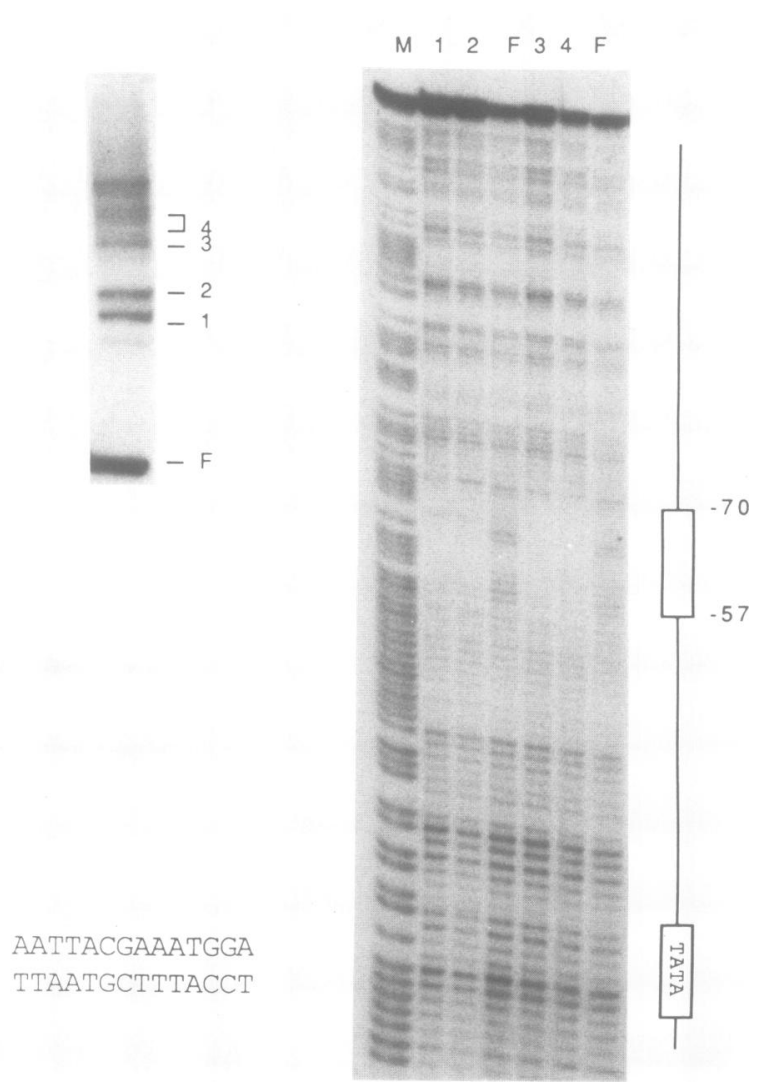

Fig. 4. Footprints obtained with phenanthroline copper treatment of the DNA-protein complexes on the gel matrix. The probes were incubated with nuclear extract from IL-6-treated Hep3B cells in the presence of salmon sperm DNA. In panel A the Hp A fragment was used as probe. $\mathrm{F}$ indicates the free DNA, M indicates the $G+A$ reaction obtained from the same probe. The retarded complexes are indicated as in Figure 3 . The protected bases are indicated. In panel $\mathrm{B}$ the $\mathrm{Hp}$ fragment $\mathrm{D}$ was used as probe.

relatively confined sequence alterations (mutations -157 and -61) prevent all the interaction observed by gel shift experiments. Further information on the characteristics of the complexes 1-5 was obtained by the analysis of footprints of the various complexes. For this purpose we performed a cleavage reaction in the gel matrix using 1,10-phenanthroline copper. This assay allows the definition of the protected DNA sequence in each specific retarded band (Kuwabara and Sigman, 1987). The gel shift experiment was performed with the indicated DNA segments, in the presence of salmon sperm DNA in order to eliminate all complexes at site B (see Materials and methods for details). As shown in Figure 4A all the different retarded bands formed at site A show a protected region from position -171 to -157 . The complexes formed at site $\mathrm{C}$ (see Figure 1) yield identical footprints for all complexes from position -70 to -57 . As expected the sequences altered in $M-61$ and $M-157$ are contained within the protected regions.

\section{The oligonucleotides containing the $A$ and $C$ binding sites confer IL-6 response to a heterologous promoter in hepatoma cells}

Transcriptional analysis indicates that, in the context of the Hp promoter, the integrity of the domains $\mathrm{A}, \mathrm{B}$ and $\mathrm{C}$ is important for both uninduced expression and IL-6 induction.

On the basis of the observation that the only difference between induced and uninduced cells is the appearance of complex 4 we have adopted the working hypothesis that the IL-6-responsive element is present twice on the Hp promoter, at the $\mathrm{A}$ and the $\mathrm{C}$ domain. In order to activate transcription, the co-operation of other cis-acting elements is however necessary, as shown by the effect of M-111.

We therefore decided to analyse whether the single binding sites could respond to IL- 6 in a different promoter context. We cloned multimers of the oligonucleotides containing the A, B or C binding sites in front of the 21-bp repeats of the SV40 promoter in the pUC19-CAT plasmid (Oliviero et al., 1987) and transfected the resulting constructions (respectively, pOL-157, pOL-111 and pOL-61) in uninduced or IL-6-treated cells. The results are shown in Figure 5. Following IL-6 treatment the plasmid pUC19-CAT is slightly induced (1.5- to 2-fold). Both pOL-61 and pOL-157 transcription is 8- to 9-fold increased upon induction while pOL-111 shows only a weak response to IL-6 (Figure 5).

\section{Discussion}

The current view of the changes in the concentration of several plasma proteins during the acute phase of inflammation is that this constitutes an early response to the pathological insult, aimed at the defence of the organism and at the re-establishment of homeostasis (Fey and Fuller, 1987). Setting aside these physiological considerations, it is clear that infections or trauma cause a strong increase in 


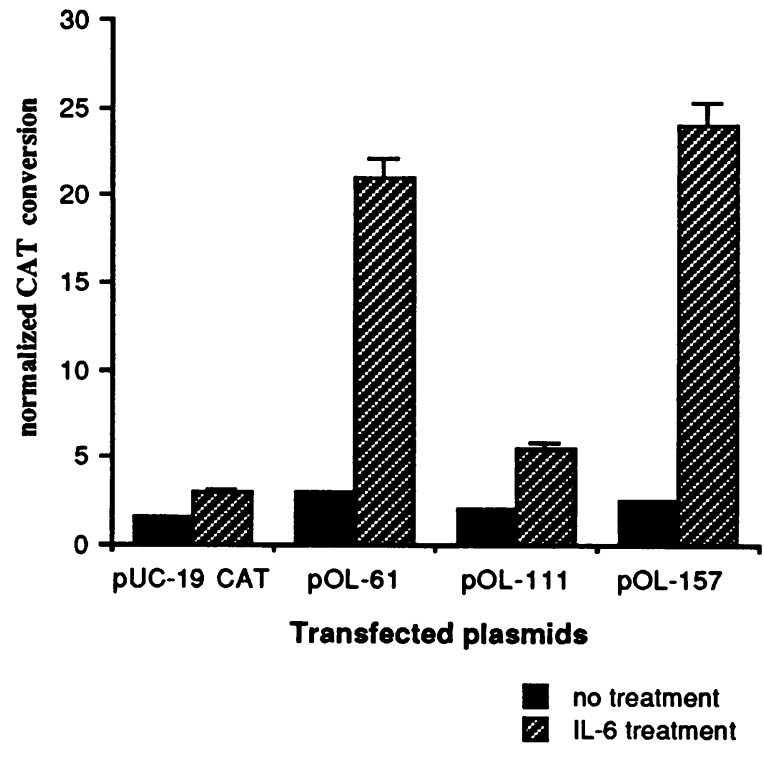

Fig. 5. Histogram indicating the CAT activity (average of two independent experiments) resulting from transfection into Hep3B of the different plasmid constructions as indicated. The expression of pUC19-CAT in unstimulated cells has been defined as 1 .

the synthesis and secretion of several factors, including IL-6. As a consequence, major albeit transient changes occur in the regulation of gene expression in the hepatocyte. IL- 6 is secreted by several cell types, and is capable of stimulating differentiation of B-lymphocytes, secretion of antibodies, as well as growth of hybridomas and plasmacytomas (Van Damme et al., 1987). It is not yet known whether all the effects of IL-6 are based on the same molecular mechanism.

The stimulation of transcription of some hepatic genes can be reproduced in a simple experimental system when cloned DNA segments transfected in hepatoma cell lines. It was therefore possible to establish that the proximal 5'-flanking promoter regions of the genes coding for haptoglobin, factor $B$ and C-reactive protein, contain IL-6-responsive elements (Morrone et al., 1988; Arcone et al., 1988). In this paper we have examined the promoter of the human haptoglobin gene in greater detail and concluded that there are three important cis-acting elements, A, B and C. A and C are both capable of conferring IL-6 inducibility to an heterologous promoter. $\mathrm{A}$ and $\mathrm{C}$ bind to the same set of proteins, implying that they contain functionally identical transcriptional signals. The results of cross-competition DNA binding experiments support this conclusion. In addition we could show that the various protein-DNA complexes formed with either A or C fragments, including the IL-6-specific complex (band 4, Figure 2) yield an identical footprint. We do not yet know whether this is due to the binding of multiple proteins which share similar DNA binding domains, to variously modified forms of the same protein, or perhaps even by association of a single DNA - protein complex with additional proteins. We think it unlikely that the multiple complexes are a consequence of artefactual proteolytic cleavage of a larger protein, because the pattern is highly reproducible and because several other DNA-binding proteins tested in the same extracts are not degraded (data not shown). What is described above corresponds also to complex 4 , which is specifically present in extracts from IL-6-induced cells. All the other complexes are present in apparently similar amounts in both extracts. This suggests that complex 4 is
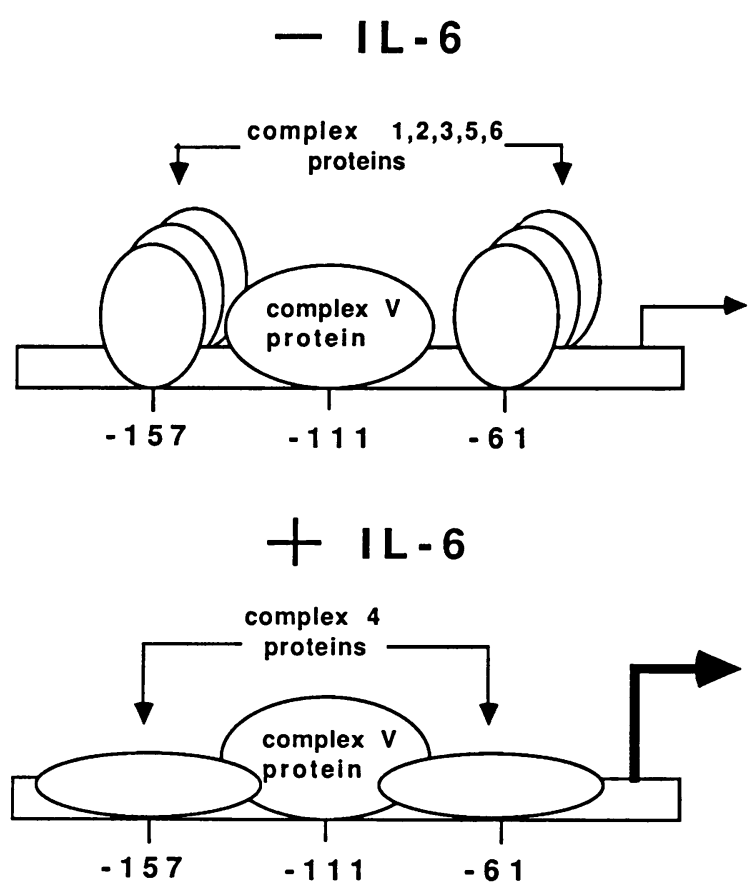

Fig. 6. Scheme illustrating the model described in the text. In the absence of IL- 6 the Hp promoter is transcribed at a low, basal level. In the presence of IL- 6 complex 5 protein is unmodified but the other proteins are substituted by complex 4 protein. This results in activation of transcription, represented by the horizontal arrows.

due to a new IL-6-dependent activity and not to the modification of a pre-existing DNA-binding protein. Inspection of the DNA sequence comprised within the footprints on fragment $\mathrm{A}$ and $\mathrm{C}$ is not particularly informative. When aligned they display the sequence GAA in equivalent positions, in the middle of the protected regions. However the mutation -157 , while causing a dramatic reduction of binding and transcription, does not involve this motif. The precise definition of the recognition site must await further experiments.

There is no IL-6-dependent change of the complexes formed with the B fragment. For this reason they were not analysed in detail. We did however establish on the basis of the results obtained with competition with wild-type and mutated oligonucleotides, that only the behaviour of complex $\mathrm{V}$ correlates with transcriptional activity. This complex is specifically competed by sequences present in the promoter of the C-reactive protein gene, also an acute-phase reactant. The significance of this observation remains to be established.

The simplest model suggested by our results is represented in Figure 6. Here we imagine that in uninduced cells the protein responsible for the formation of complex $\mathrm{V}$ is sitting on DNA flanked by any of the proteins responsible for complexes 1, 2, 3, 5 and/or 6 , bound to site $A$ and site $C$. In this conformation the gene is poorly transcribed either because some of the $\mathrm{A}$ and $\mathrm{C}$ binding proteins are repressors or because they are poor activators. Upon IL- 6 induction there is no change at the B site, but the IL-6-dependent protein, responsible for complex 4 , substitutes the other proteins on sites $\mathrm{A}$ and $\mathrm{C}$. This new conformation is active in transcription because the complex 4 protein is either a better activator or an anti-repressor. In this model full transcriptional activity is only obtained through the cooperation of the complex 4 protein and the protein binding 
at the B site. Alternative conformations are however possible, as shown by the IL-6-dependent activation of the SV40 promoter by multiple complex 4 protein binding sites. Here presumably co-operation is achieved between complex 4 protein and any of the well characterized transcriptional factors binding to the SV40 promoter, such as Spl or Apl. In this hybrid promoter the polymerized $\mathrm{Hp} \mathrm{C}$ sites act as an IL-6-dependent enhancer because equivalent activation is observed with two copies of the site $C$ in the physiological orientation (plasmid pOL-61) and with three copies of site A, all in the inverted orientation (plasmid pOL-157).

In this model the effect of IL-6 is exerted by inducing the formation (by synthesis or modification) of a new transcriptional factor, which in turn activates IL-6-responsive genes. It will be interesting to establish whether this is a hepatocyte-specific phenomenon, as some preliminary experiments indicate, because complex 4 protein is not observed in IL-6-treated HeLa cells (V.Poli, unpublished data) or whether the same DNA-binding protein is induced in other IL-6-responsive systems.

\section{Materials and methods}

\section{Cell culture, DNA transfections and CAT assays}

The human hepatoma cell line Hep3B (Knowles et al., 1981) and HeLa cells were cultured as monolayers in Dulbecco's modified Eagle's medium supplemented with $10 \%$ fetal calf serum.

Human recombinant IL-6 was prepared using recombinant vaccinia virus as described (Stunnenberg et al., 1988). Its activity, assayed as growthpromoting activity for the hybridoma 7TD1 cells, was $2 \times 10^{5}$ units $/ \mathrm{ml}$ Induced cells were treated for $40 \mathrm{~h}$ with the recombinant IL- 6 at the concentration of $500 \mathrm{U} / \mathrm{ml}$.

DNA transfections were performed by the calcium phosphate precipitation technique (Graham and van der Eb, 1973). Cells were transfected with $10 \mu \mathrm{g}$ (5 $\mathrm{cm}$ dishes) or $20 \mu \mathrm{g}$ (10 cm dishes) of plasmid DNA; each precipitate contained the plasmid as an internal marker at $1 / 20$ th of the total DNA. Transfected cells were harvested $48 \mathrm{~h}$ after transfection measuring both for CAT and luciferase activity. The CAT assays were performed according to Gorman et al. (1982). The quantitation was done by cutting the area of the chromatogram corresponding to the acetylated and non-acetylated forms of the $\left[{ }^{14} \mathrm{C}\right]$ chloramphenicol and counting them in a liquid scintillation counter. The ratio of the acetylated form to the total was then normalized by comparison with the luciferase activity measured as described (DeWet et al., 1987).

\section{Site-directed mutagenesis and plasmid constructions}

The mutagenesis of the -184 to +30 region of the $\mathrm{Hp}$ in the plasmid pEMBL8-CAT was carried out as described (De Simone et al., 1987). The single strand of Hp186-CAT was used as a template for the in vitro synthesis of the complementary strand. A set of synthetic oligonucleotides containing the base substutions to be inserted (flanked at both sides by 10 matching nucleotides) were used as primers for the second strand. The mutations were designed to insert a site for the $E c o \mathrm{RV}$ restriction enzyme. Mutant clones were verified by sequencing.

The single-stranded oligonucleotides OL-61, OL-111 and OL-157 (see Table I for the sequences) were polymerized by ligation, gel purified and inserted in the BamHI site of the pUC19-CAT. The plasmid pOL-61 contains two copies of the OL-61 oligonucleotide in the physiological orientation. The plasmid pOL-111 contains three copies of the OL-111 oligonucleotide, two in the physiological orientation and the middle ones in the inverted orientation. The plasmid pOL-157 contains three copies of the OL-157 oligonucleotide, all three in the non-physiological orientation.

\section{Cell protein extracts and gel retardation assay}

Protein extracts from cultured cell nuclei were prepared according to Shapiro et al. (1988).

The end-labelled probes were prepared from Hp-CAT (Oliviero $e t$ al., 1987) construction either by BamHI/SacI (probe A), or by SacI/HindIII (probe D) digestion and labelled by Klenow polymerase with $\left[\alpha-{ }^{32} \mathrm{P}\right] \mathrm{dATP}$ and $\left[\alpha^{-32} \mathrm{P}\right] \mathrm{dCTp}$. The B probe has been obtained from the plasmid HpM-87 by $S a c \mathrm{I} / E c o \mathrm{RV}$ and end labelled with $\left[\gamma^{32} \mathrm{P}\right] \mathrm{dATP}$ by T4 polynucleotide kinase. The double-stranded oligonucleotides used were labelled with the T4 polynucleotide kinase in the presence of $\left[\gamma^{-32} \mathrm{P}\right]$ ATP. Briefly, individual bands were excised from the band-shift gels and subjected to nuclease digestion within the acrylamide matrix with $4 \mathrm{mM}$ 1,10-phenantroline-copper ion, $1.45 \mathrm{mM} \mathrm{CuSO}_{4}$ and 3-mercaptopropionic acid. The reactions were quenched by adding 2,9-dimetic onto phenontroline to the reaction mixture. The samples were subsequently eluted from the acrylamide matrix and run on a $6 \%$ acrylamide denaturing gel.

Gel retardation assays were carried out according to Fried and Crothers (1983), nuclease experiments in the gel matrix were performed according to Kuwabara and Sigman (1987).

\section{Acknowledgements}

We wish to thank R.Di Lauro, G.Grimaldi and I.Mattaj for critical reading of the manuscript and V.Poli for sharing her unpublished results. S.O. was the recipient of an EMBO fellowship. This work was partially supported by a grant from the EEC (BAP-0115-D).

\section{References}

Arcone,R., Gualandi,G. and Ciliberto,G. (1988) Nucleic Acids Res., 16. 3195-3207.

Baumann,H., Firestone,C.L., Burgess,T.L., Gross,K.W., Yamamoto,K.R. and Held,W.A. (1983a) J. Biol. Chem., 258, 563-570.

Baumann,H., Jahreis,G.P. and Gaines,K.C. (1983b) J. Cell Biol., 97, 866-876.

Baumann,H., Jahreis,G.P., Sauder,D.N. and Koj,A. (1984) J. Biol. Chem., 259, $7331-7342$.

Baumann,H., Hill,R.E., Sauder,D.N. and Jahreis,G.P. (1986) J. Cell Biol., 102, 370-383.

Baumann,H., Onorato,V., Gauldie,J. and Jahreis,G.P. (1987a) J. Biol. Chem., 262, 9756-9768.

Baumann,H., Richards,C. and Gauldie,J. (1987b) J. Immunol., 139, 4122-4128.

Billiau,A. (1987) Immunol. Today, 8, 84-87.

Ciliberto,G. (1988) In Proceedings of the XIII Argenteuil Symposium Acute Phase Proteins and Acute Phase Response. Brussels, Belgium, in press. Darlington,G.J., Wilson,D.R. and Lachman,L.B. (1986) J. Cell Biol., 103, 787-793.

De Simone,V. and Cortese, R. (1988) In MacLean,N. (ed.), Oxford Surveys of Eukaryotic Genes. Oxford University Press, Oxford, Vol. 5, pp. $51-90$.

De Simone,V., Ciliberto,G., Hardon,E., Paonessa,G., Palla,F., Lundberg, L. and Cortese,R. (1987) Embo J., 6, 2759-2766.

DeWet,J.R., Wood,K.V., DeLuca,M., Helsinki,D.R. and Subramani,S. (1987) Mol. Cell. Biol., 7, 725-737.

Fey,G.H. and Gauldie,J. (1989) In Popper,H. and Shappuer,F. (eds), Progress in Liver Disease. Grune and Stratten, Philadelphia, PA, in press. Fey,G.H. and Fuller,G.M. (1987) Mol. Biol. Med., 4, 323-338.

Fried,M. and Crothers,D.M. (1981) Nucleic Acids Res., 9, 6505-6525.

Gauldie,J., Richards,C., Harnish,D., Lansdorp,P. and Baumann,H. (1987) Proc. Natl. Acad. Sci. USA, 84, 7251-7255.

Gorman,C.M., Moffat,L.F. and Howard,B.H. (1982) Mol. Cell. Biol., 2, $1044-1051$.

Graham,F.L. and van der Eb,A.J. (1973) Virology, 52, 456-467.

Kishimoto, T. and Hirano, T. (1988) Annu. Rev. Immunol., 6, 485-512.

Koj,A. (1987) In Reutter,?. (ed.), Modulation of Liver Cell Expression. Falk Symposium No. 43, MTP Press Ltd, pp. 331-342.

Koj,A., Gauldie,J., Regoeczi,E., Sander,D.N. and Sweehey,G.H. (1984) Biochem. J., 22, 550-514.

Knowles,B.B., Howe,C.C. and Aden,D.P. (1981) Science, 209, 497-499.

Kushner,I. (1982) Ann. N.Y. Acad. Sci., 389, 39-48.

Kuwabara,M.D. and Sigman,D.S. (1987) Biochemistry, 26, 7234-7238.

Morrone,G., Ciliberto,G., Oliviero,S., Arcone,R., Dente,L., Content,J. and Cortese,R. (1988) J. Biol. Chem., 263, 12554-12558.

Oliviero,S., Morrone,G. and Cortese,R. (1987) EMBO J., 6, 1905- 1912.

Paonessa,G., Gounari,F., Frank,R. and Cortese,R. (1988) EMBO J., 7, $3115-3123$.

Perlmutter,D.H., Goldberger,G., Dinarello,C.A., Mizel,S.B. and Colten, H.R. (1986) Science, 32, 850-852.

Ptashne,M. (1988) Nature, 335, 683-689.

Ramadori,G., Sipe,J.D., Dinarello,C.A., Mizel,S.B. and Colten,H.R. (1985) J. Exp. Med., 162, 930-942.

Seghal,P.B., May,L.T., Tamm,I. and Vilcek,J. (1987) Science, 235, $731-732$. 
Shapiro,D.J., Sharp.P.A., Waly,W.W. and Keller,M.J. (1988) DNA, 7. $47-55$.

Stunnenberg.H.G., Lange.H., Philipson.L., van Miltenburg.R. and van der Vliet,P. (1988) Nucleic Acids Res., 16, 2431-2444.

Van Damme,J., Opdenakker,G., Simpson,R.J., Rubira,M.R., Cayphas,S., Vink,A., Bilian,A. and Van Snick,J. (1987) J. Exp. Med., 165, 914-919. Woloski,B.M. and Fuller,G.M. (1985) Proc. Natl. Acad. Sci. USA, 82, $1443-1447$

Wong,G.G. and Clark,S.C. (1988) Immunol. Today, 9, 137-139.

Zilberstein,A., Ruggieri,R., Korn.J.N. and Revel,M. (1986) EMBO J., 5, 2529-2537.

Received on November 10, 1988; revised on January 12, 1989 